\title{
DEVELOPMENT AND APPLICATION OF WIDE BANDWIDTH MAGNETO-RESISTIVE SENSOR BASED EDDY CURRENT PROBE
}

\author{
Buzz Wincheski $^{1}$ and John Simpson ${ }^{2}$ \\ ${ }^{1}$ NASA Langley Research Center, Hampton, VA 23681 \\ ${ }^{2}$ Lockheed Martin Space Operations, Hampton, VA 23681
}

\begin{abstract}
The integration of magneto-resistive sensors into eddy current probes can significantly expand the capabilities of conventional eddy current nondestructive evaluation techniques. The room temperature solid-state sensors have typical bandwidths in the megahertz range and resolutions of tens of microgauss. The low frequency sensitivity of magneto-resistive sensors has been capitalized upon in previous research to fabricate very low frequency eddy current sensors for deep flaw detection in multilayer conductors. In this work a modified probe design is presented to expand the capabilities of the device. The new probe design incorporates a dual induction source enabling operation from low frequency deep flaw detection to high frequency high resolution near surface material characterization. Applications of the probe for the detection of localized near surface conductivity anomalies are presented. Finite element modeling of the probe is shown to be in good agreement with experimental measurements.
\end{abstract}

Keywords: Eddy Current, Magneto-resistive, high frequency, high resolution PACS: 81.70.Ex, 81.40.Np, 81.05.Bx

\section{INTRODUCTION}

The use of magneto-resistive sensors in the fabrication of novel eddy current probes has been performed by several groups over the last decade [1-5]. The focus of these efforts has been the development of sensors that can operate at very low frequencies without a loss of sensitivity to the magnetic field, leading to the development of systems with flaw detection capabilities at centimeter-scale flaw depths [1-2]. In this work the high frequency capabilities and small spatial size of typical magneto-resistive sensors is explored for the development of high resolution near surface material characterization. Commercial, offthe-shelf, anisotropic magneto-resistive sensors are employed as the sensing element in the probe. The sensors operation from DC to megahertz frequencies enables the configuration of a wide bandwidth eddy current probe. Optimization of the probe for both deep flaw detection and near surface material characterization is performed using a dual induction source tailored for operation in each of the two regimes. 


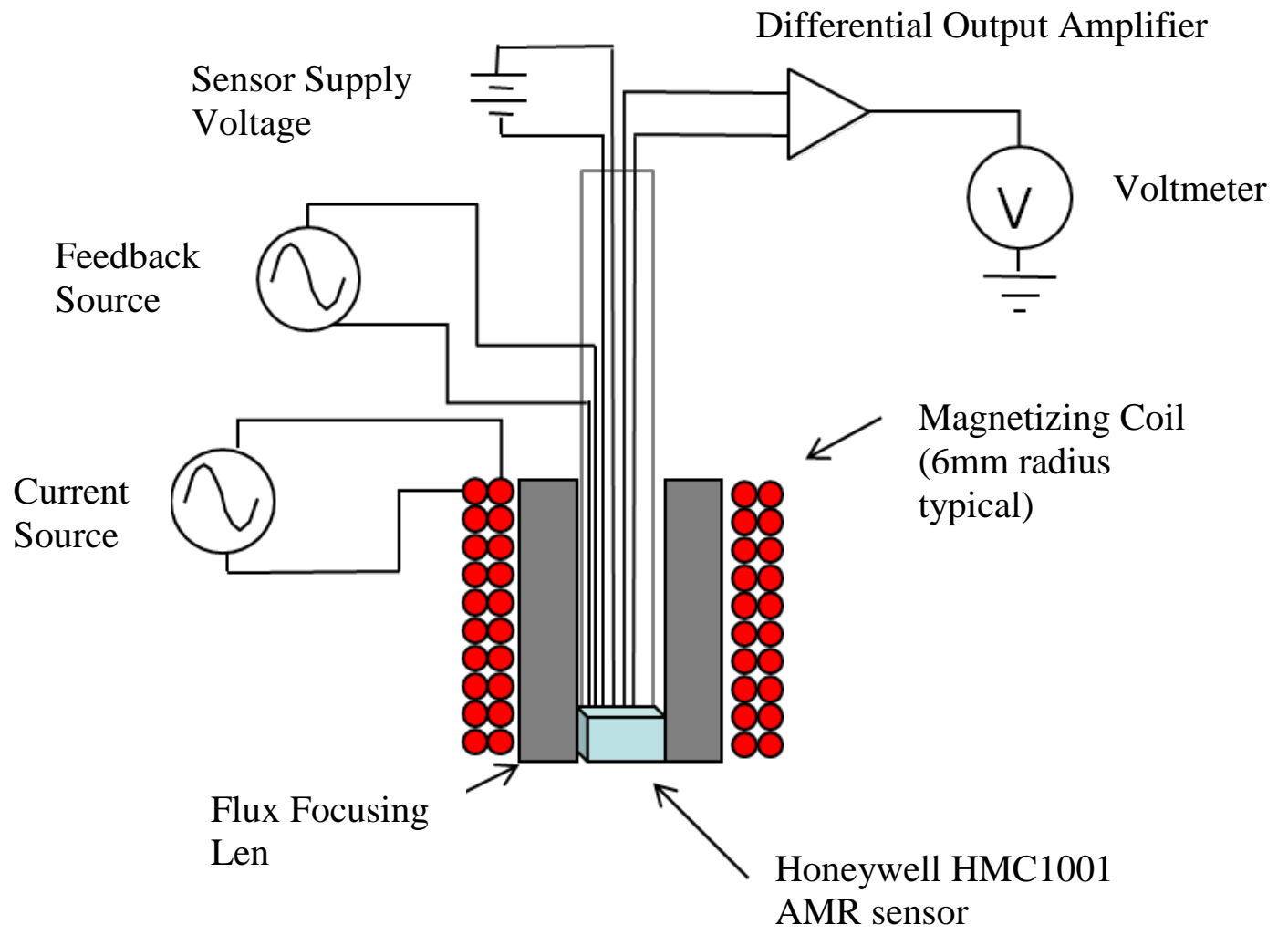

FIGURE 1. Magneto-resistive flux focusing eddy current probe.

\section{MAGNETO-RESISTIVE FLUX FOCUSING EDDY CURRENT PROBE}

The design of the wide bandwidth magneto-resistive sensor was conceived based upon adding high resolution near surface material characterization capabilities to the magneto-resistive flux focusing eddy current probe [1,5]. A schematic diagram of the probe, developed for deep flaw detection in multi-layer structures, is shown in figure 1. The unique probe design incorporates a driver pickup configuration in which a mu-metal flux-focusing lens is used to minimize direct coupling between the drive coil and the magnetic field sensor. When placed above a material under test, the alternating magnetic field of the magnetizing coil induces a current flow that diffuses into the test object. The probe is designed such that the magnetic field along the centerline of the probe decays slowly with depth into the material under test, enabling deep flaw detection in multi-layer structures. The spatial resolution of the probe, however, is limited by the relatively large diameter of the induction source and the diffusive properties of the low frequency induced currents into the part under test.

\section{DESIGN OF WIDE BANDWIDTH MAGNETO-RESISTIVE SENSOR BASED EDDY CURRENT PROBE}

In designing a magneto-resistive probe capable of achieving high spatial resolution it is noted that the limiting factors on the spatial resolution of the previous design, discussed above, are solely attributable to the induction source. The AMR sensing element itself is quite small, with $\mathrm{X}$-ray imaging of the sensor chip showing the surface area of the thin film AMR bridge to be approximately $0.7 \mathrm{~mm}^{\wedge} 2$, and the wide bandwidth of the sensors 

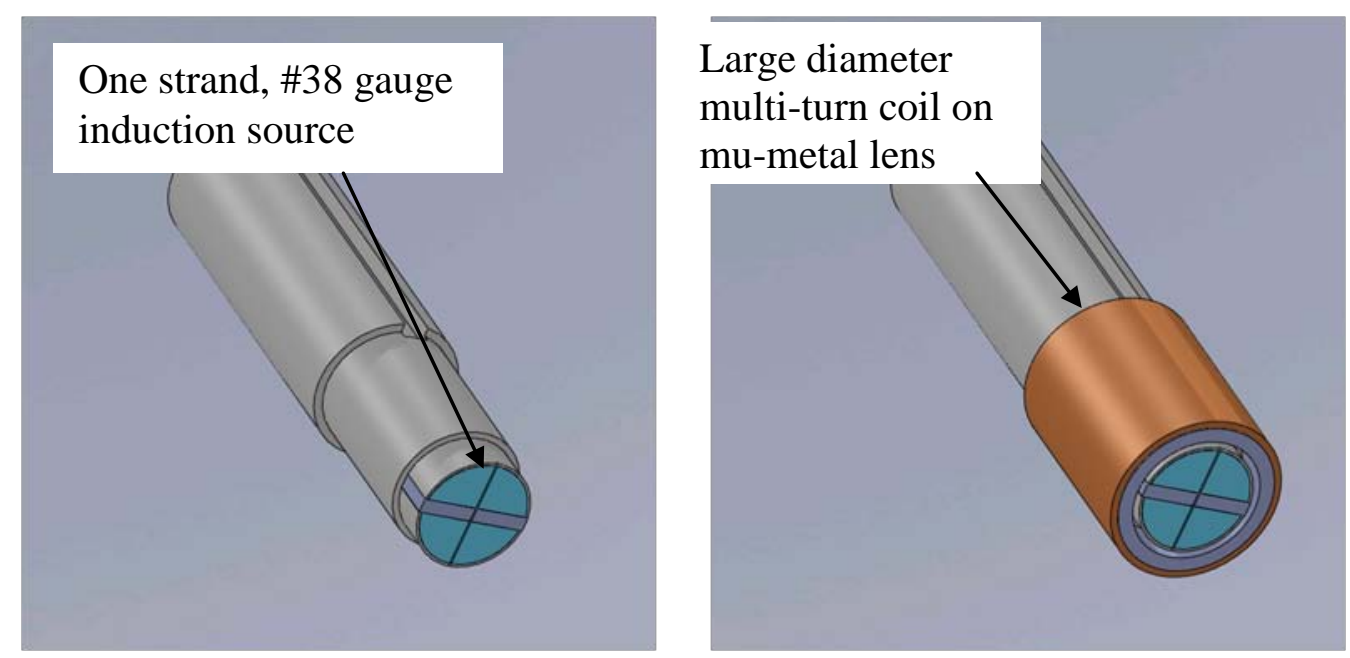

FIGURE 2. Design of AMR Eddy Current Probe for deep inspection capabilities with high spatial resolution for surface breaking flaws.

enable operation from DC up to $5 \mathrm{MHz}$ [6]. Design changes for high spatial resolution must therefore focus on the induction source.

Figure 2 displays the incorporated design change to the induction source of the magneto-resistive sensor based eddy current probe to enable both high resolution, near surface imaging and deep flaw detection capabilities. A second induction source specifically for high frequency, high spatial resolution imaging has been added to the probe design. The induction source consists of a single strand copper wire (\#38 Gauge, 100 micron diameter) placed directly across the face of the probe. The wire is positioned to produce maximum coupling between the field lines of the wire and the AMR sensor bridge. The sensitive axis of the AMR bridge is aligned with the centerline of the probe. The wire is recessed into the surface of the probe (and the AMR sensor chip) to avoid damage to the wire during inspections. Low frequency deep flaw detection capabilities are maintained with the original large diameter multi-turn coil wound on a mu-metal lens. The large difference in operating frequency of the coils enables simultaneous operation of the probe for both high frequency, high resolution imaging of surface features and low frequency, deep flaw detection. Typical operating frequencies in the two regimes are $1 \mathrm{MHz}$ and 500 $\mathrm{Hz}$ respectively.

\section{FINITE ELEMENT MODELING RESULTS}

Three dimensional finite element modeling for a simplified inspection geometry was performed to test the feasibility of the probe design shown in fig. 2. All finite element modeling results were acquired using the COMSOL multi-physics AC/DC package. As shown in fig. 3 , the model consists of a single strand induction source above a conducting plate with a 500 micron diameter x 250 micron deep flat-bottom-hole. The complete discretized volume is $100 \mathrm{~mm}^{\wedge} 3$ and consists of roughly 300000 elements. An external current density of $2 \mathrm{e} 6 \mathrm{~A} / \mathrm{m}^{\wedge} 2$ at a frequency of $1 \mathrm{MHz}$ was placed in the induction wire, equivalent to $16 \mathrm{~mA}$ total current in the 100 micron diameter wire.

The modeling data was processed to extract the magnetic field in the vertical direction (normal to the surface of the part under test) at the sensor location for various hole to induction wire distances, with the position of the sensor held constant relative to the induction wire. In all runs the induction wire was placed parallel to the y axis through the point $\mathrm{x}=0, \mathrm{z}=120$ microns. The hole to induction wire distance was changed by displacing the center of the hole along the $\mathrm{x}$ axis at $\mathrm{y}=0$. The measurable magnetic field at each hole 


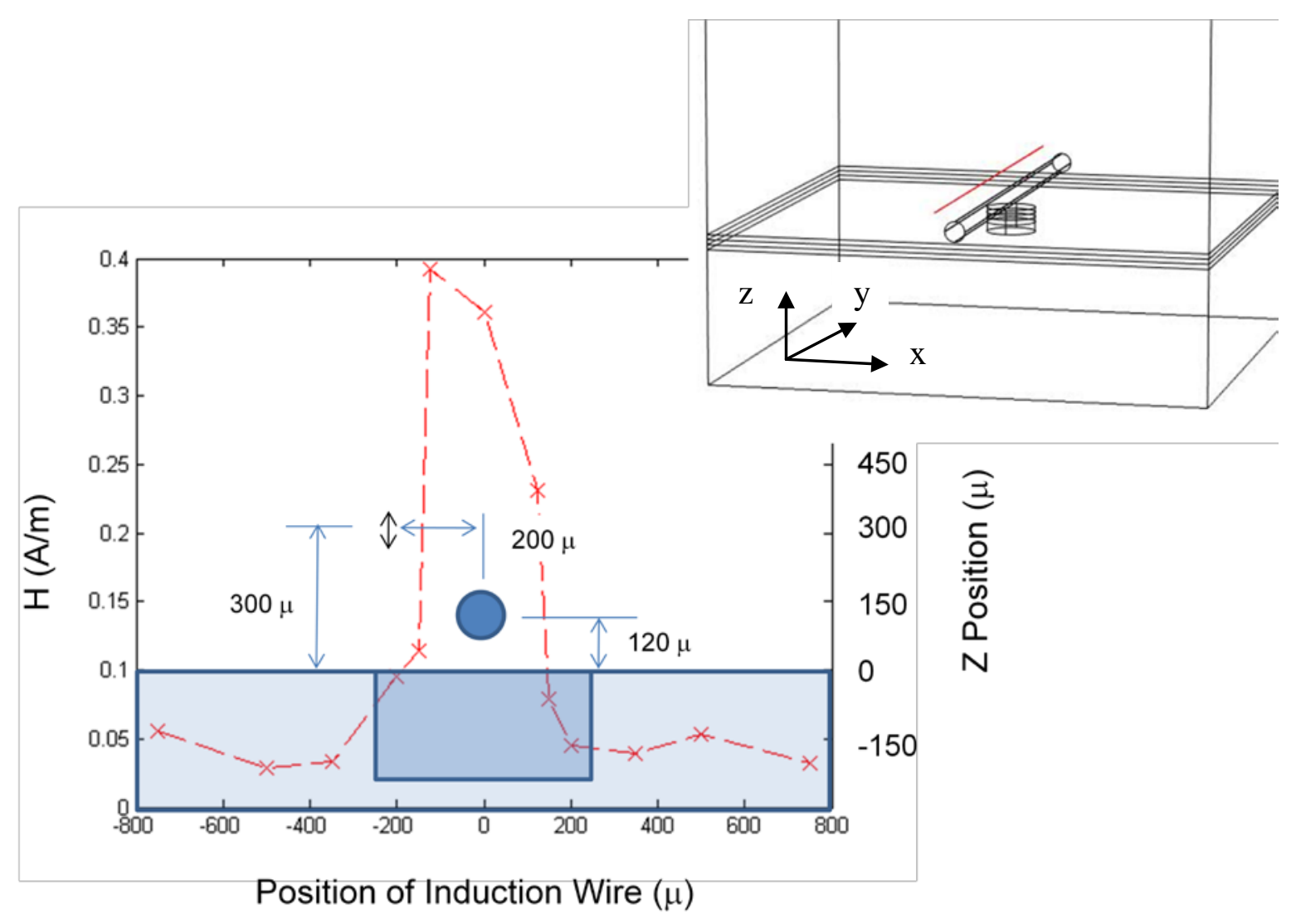

FIGURE 3. Finite element model and results for simplified inspection geometry.

to wire distance was calculated by extracting the field values along a line parallel to the induction source and displaced in the $(x, z)$ plane a distance equivalent to the targeted spacing between the induction wire and AMR sensor in the eddy current probe. The difference in the field between the $\mathrm{y}=0$ position (aligned with the hole center) and the $\mathrm{y}=2 \mathrm{~mm}$ position (null location 'far' from hole) was taken as the change in the magnetic field at the sensor location due to the flat-bottom-hole. These results are displayed in the chart in Fig. 3. Overlaid on the chart is a cross sectional view of the geometry corresponding to the induction wire location centered on the flat bottom hole. The sensor location and sensitive axis is represented on the graph by the double arrow just above and to the left of the induction wire. The line graph displays the field change as the sensor and induction wire are scanned across the flat-bottom-hole. A peak field change of approximately $0.4 \mathrm{~A} / \mathrm{m}$ (5 mOe) is predicted, more than two orders of magnitude above the guaranteed resolution of the sensors [6]. The line graph shows an asymmetry about the center of the hole due to the fixed location of the sensor to the left hand side of the induction wire.

\section{EXPERIMENTAL RESULTS}

Experimental evaluation of the high frequency capabilities of the wide bandwidth magneto-resistive sensor based eddy current probe was performed using a reference standard fabricated to the specifications shown in Fig. 4. The reference standard was constructed from a 1/4" thick aluminum 2024 plate. A series of drills were machined into the part at varying diameter, depth, and center to center distances in order to evaluate the surface breaking flaw detection capabilities and the spatial resolution of the probe. The smallest drill size, depth, and center to center distances were .0135 ”, .01”, and .025” respectively. 


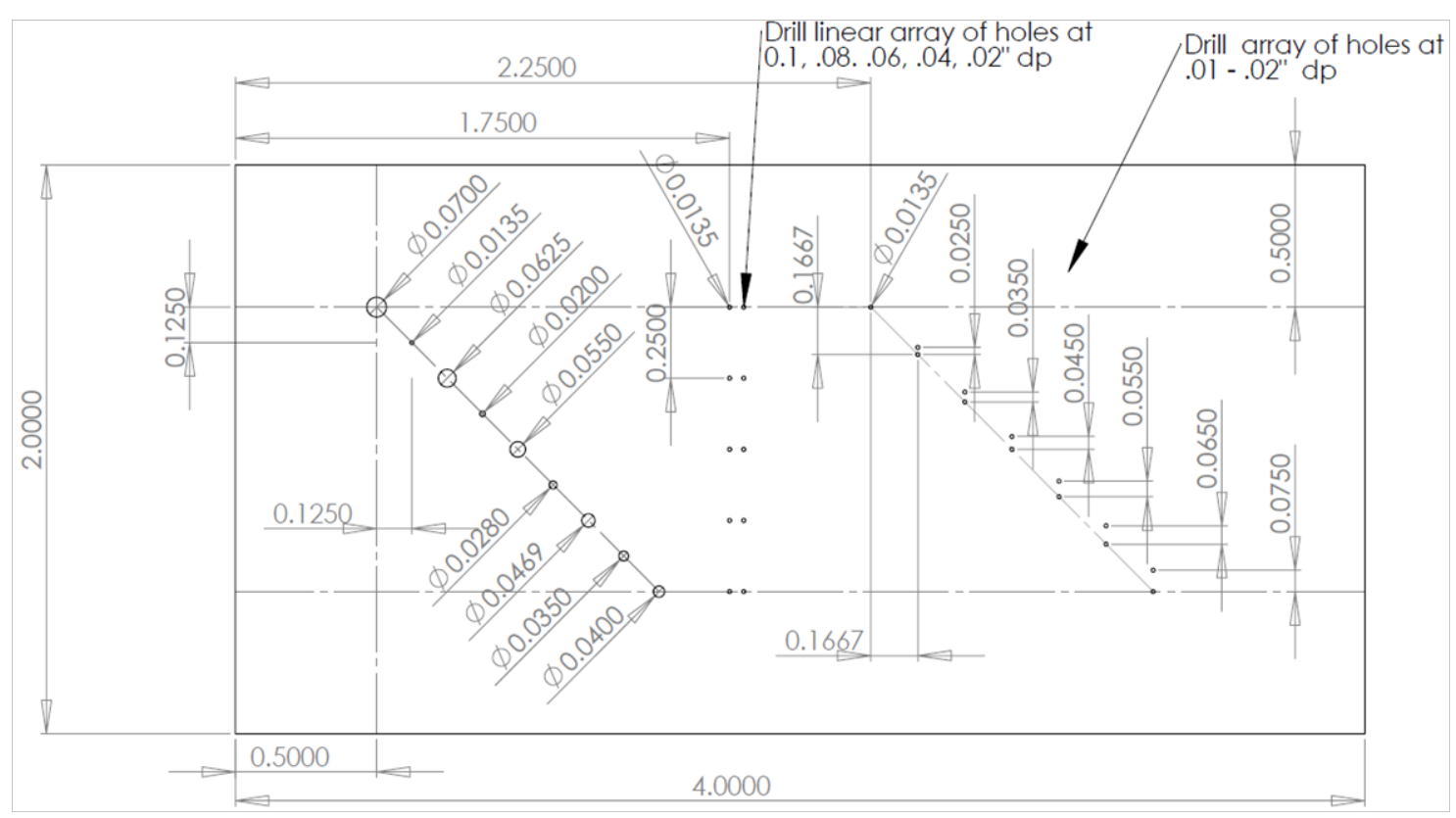

FIGURE 4. Design of test standard for experimental evaluation of wide bandwidth magneto-resistive sensor based eddy current probe.

The wide bandwidth magneto-resistive sensor based eddy current probe was constructed based upon the design shown in Fig. 2. The probe face diameter is $10 \mathrm{~mm}$, excluding the mu-metal lens and large diameter multi-turn coil. For the high frequency evaluation, the sensor and induction wire were covered with a mu-metal shield with outside diameter of $13.5 \mathrm{~mm}$ and height of $19 \mathrm{~mm}$. Although the large diameter multi-turn coil was not included in this round of testing, it would be wound directly on this mu-metal lens as shown in Fig. 2.

The eddy current probe was driven at a frequency of $1 \mathrm{MHz}$ while the output of the AMR sensor was monitored using a lock-in amplifier with a band pass filter referenced to the drive signal. Data were acquired by digitizing the amplitude and phase outputs of the lock-in amplifier and storing these values as a function of position as the part was scanned under motion control. Before beginning a scan, the outputs of the lock-in amplifier were zeroed on an unflawed portion of the sample using the built in auto-offset function of the device. In order to enable high spatial resolution the time constant of the lock-in amplifier was reduced to $20 \mathrm{mS}$ and the scan rate of the probe over the part was set at $3 \mathrm{~mm} / \mathrm{second}$. During a scan line data were acquired every $40 \mathrm{mS}$, twice as long as the output filter time constant, producing a grid spacing of 120 microns.

Fig. 5 displays two C-scans for the inspection of the calibration standard of Fig. 4 with the wide bandwidth magneto-resistive sensor based eddy current probe operating at 1 MHz. The plotted data is the normalized amplitude of the AMR sensor which is proportional to the increase in the magnetic field amplitude in the $\mathrm{z}$ direction (out of plane) as compared to an unflawed area on the sample. The amplitude data in all C-scans are plotted in logarithmic scale and cover six orders of magnitude. The direction of the induction wire is rotated between the two C-scans of Fig. 5. In Fig. 5a the induction wire is parallel to the short edge of the sample where as for Fig. 5b it is parallel to the long edge of the sample. It is clear from the figures that spatial resolution of the probe is reduced along the axis parallel to the induction wire, as the point-like indications become skewed in this direction. Scans in both directions clearly isolate the solitary .0135" diameter x .01" deep drill (the inset line plot shows the magnetic field change across this defect for the parallel orientation C-Scan). Drills spaced closer than approximately 0.05”, however, cannot be 

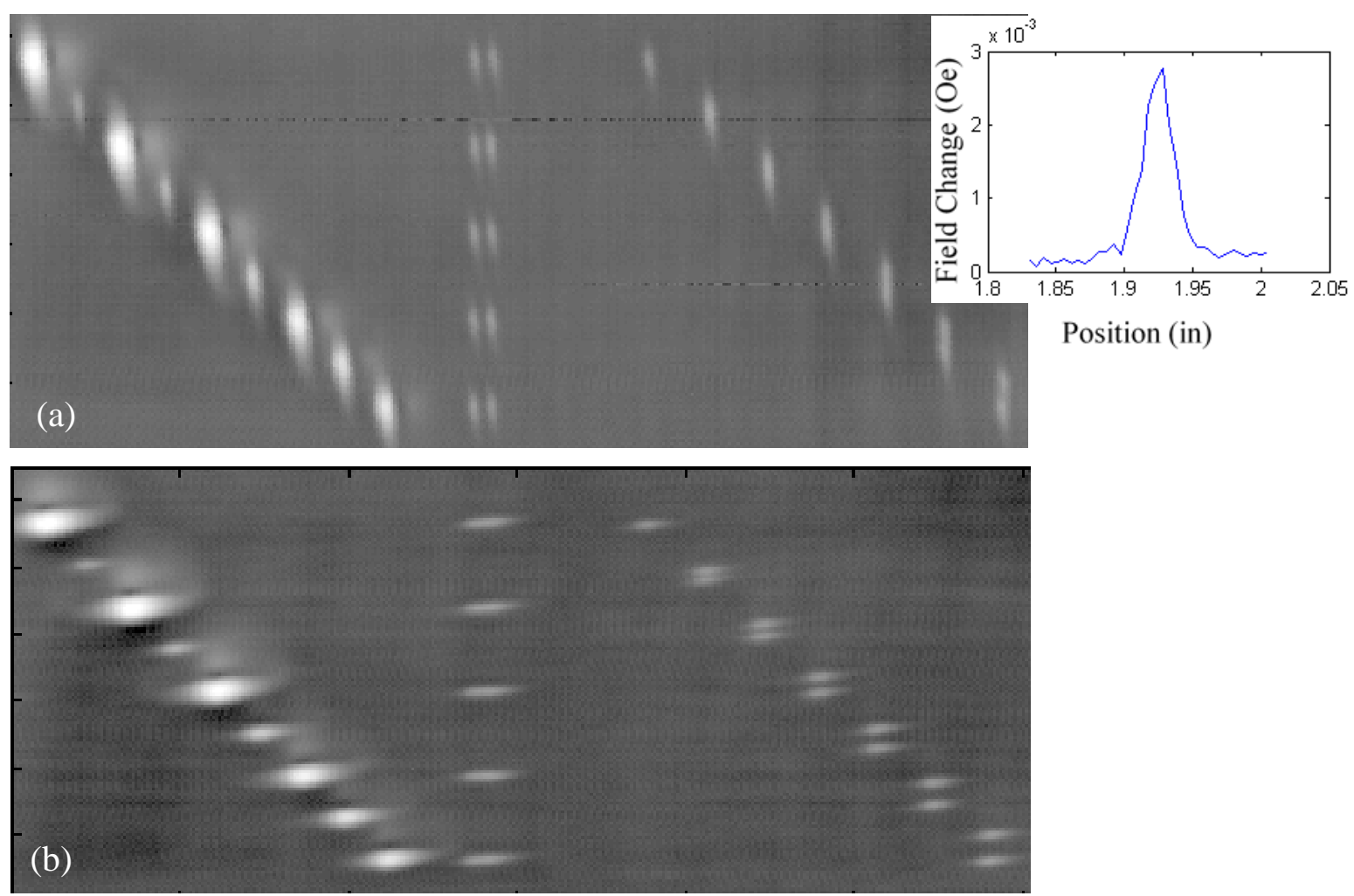

FIGURE 5. Experimental results for scan of calibration sample of Fig. 3 with Wide Bandwidth MagnetoResistive Sensor Based Eddy Current Probe where induction wire is positioned parallel to (a) short edge of sample and (b) long edge of sample. Inset line scan shows response across isolated .0135” diameter drill.

clearly individually identified when the induction wire direction is parallel to the displacement vector between the drill centers. When the induction wire direction is perpendicular to this displacement vector, even the most closely spaced holes, with 0.025 ” center to center spacing, are clearly isolated.

As the direction of the induction wire is known a-priori, it is a simple matter to reduce the spread of the data in this direction through a deconvolution of the data with the point spread function of the drive source. The point spread function of the source can be determined by examining the probe response to the isolated .0135" drill in a calibration scan. Taking this as a point defect, the spread of the C-scan data is then the point spread function of the induction source. In processing the scan data for the part under test, the predetermined point spread function is rotated to coincide with the direction of the induction wire during the scan and then deconvolved with the acquired image data. Fig. 6a displays the results of this processing for a scan in which the induction wire is placed approximately 45 degrees to each edge of the sample. The skew of the indications along the direction of the induction wire has been greatly reduced and all indications are individually resolved. For comparison with a conventional eddy current probe, Fig 6b displays the vertical channel C-scan acquired with a $1 \mathrm{MHz}$ shielded 1/8” diameter eddy current pencil probe. Here the relatively large diameter of the conventional probe results in significant overlap of the response from the individual drills. There is also very little change in the spatial area of the response for the various diameter drills. Only the magnitude of the probe response is directly related to the flaw diameter. In contrast, the high frequency component of the wide bandwidth magneto-resistive sensor based eddy current probe produces much more localized indications with both the spatial area and magnitude proportional to the drill diameter. 

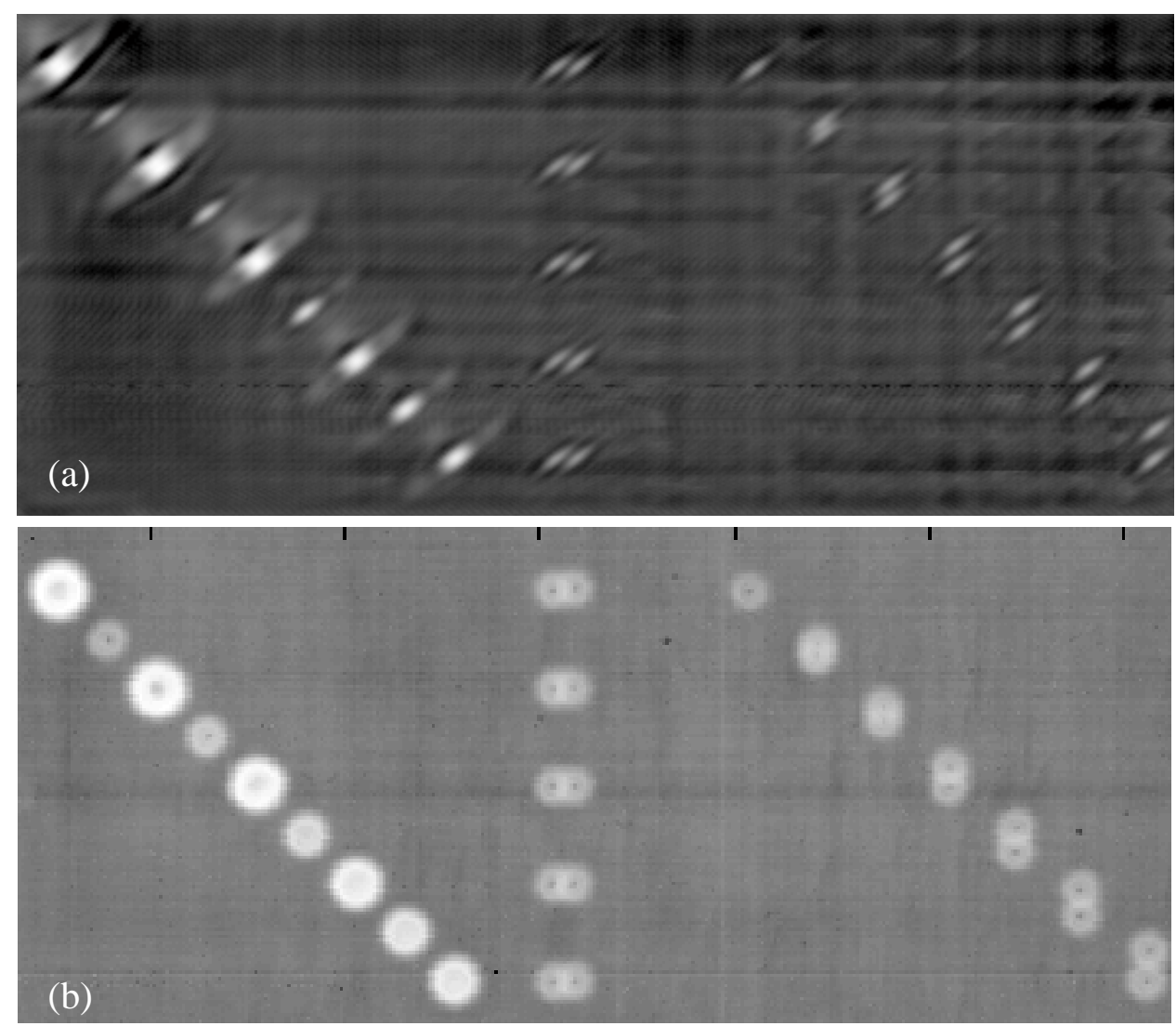

FIGURE 6. Experimental results for scan of calibration sample of Fig. 4 with (a) wide bandwidth magnetoresistive sensor based eddy current probe and (b) conventional shielded pencil probe.

\section{SUMMARY}

In this work a new eddy current probe design has been presented. The probe configuration includes two induction sources with a single pickup sensor. The use of a magneto-resistive device as the magnetic field sensor enables efficient monitoring of magnetic fields at frequencies from DC to megahertz. While the low frequencies capabilities of magneto-resisitive sensor based eddy current probes have been presented in the past [1-5], the current design adds capabilities for high frequency high spatial resolution of surface breaking defects. Along with the probe design, finite element modeling and experimental verification of the high frequency operation of the device has been presented. The results show a significant increase in the resolvability of small and closely spaced surface anomalies as compared to a conventional eddy current pencil probe, and pave the way for fabrication and testing of a wide bandwidth probe with dual induction sources for simultaneous low frequency deep flaw detection and high frequency high resolution near surface material characterization.

\section{REFERENCES}

1. B. Wincheski and M. Namkung, "Deep Flaw Detection with Giant Magnetoresisitve (GMR) based Self-Nulling Probe," in Review of progress in quantitative NDE, , Vol. 19, pp. 465-472, 2000. 
2. R. Sikora, T. Chady, S. Gratkowski, M. Komorowski, and K. Stawicki, "Eddy Current Testing of Thick Aluminum Plates with Hidden Cracks," in Review of progress in quantitative NDE, Vol. 22,pp. 427-434, 2003.

3. R. Rempt, "Using Magnetoresistive Sensors for a Sensitivity Quantum Leap in Aircraft Nondestructive Evaluation (NDE),” Proc. of SPIE,Vol. 5770, pp 32-45, 2005.

4. A. Jander, C. Smith, and R. Schneider, 'Magnetoresistive Sensors for Nondestructive Evaluation,” Presented at 10 ${ }^{\text {th }}$ SPIE Conference 5770, NVE, 2005.

5. R. Wincheski, M. Namkung, and J. Simpson, "Magnetoresistive Flux Focusing Eddy Current Flaw Detection,” U.S. Patent Number 6,888,346 B2, 2005.

6. Honeywell Sensor Products, 1- and 2-axis Magnetic Sensors, MHC1001/1002 HMC1021/1022, www.magneticsensors.com, 900248 Rev. B. 